\title{
Extended right upper sleeve lobectomy with double-barrel segmental bronchoplasty
}

\author{
Atsushi Hata, Hironobu Wada, Yuichi Sakairi, Hajime Tamura, Taiki Fujiwara, Hidemi Suzuki, Takahiro \\ Nakajima, Masako Chiyo, Ichiro Yoshino \\ Department of General Thoracic Surgery, Graduate School of Medicine, Chiba University, Chiba, 260-8670, Japan \\ Correspondence to: Hironobu Wada, MD, PhD. Assistant Professor, Department of General Thoracic Surgery, Graduate School of Medicine, Chiba \\ University, 1-8-1 Inohana, chuo-ku, Chiba, 260-8670, Japan. Email: Hironobu.Wada@chiba-u.jp.
}

Submitted Jan 16, 2018. Accepted for publication Apr 19, 2018.

doi: $10.21037 /$ jtd.2018.04.135

View this article at: http://dx.doi.org/10.21037/jtd.2018.04.135

\section{Introduction}

Extended sleeve lobectomy (ESL), which is defined as the atypical sleeve resection of more than one lobe, has been widely accepted to preserve the pulmonary function (1-3). Double-barrel anastomosis has been reported as a method of bronchial reconstruction after carinal resection; however, it is rarely applied in the reconstruction of segmental bronchi. We herein report a case in which ESL with double-barrel anastomosis of the middle lobar bronchus (MLB) and basilar bronchus (BB) was successfully applied in the treatment of a patient with centrally located lung adenocarcinoma.

\section{Operative techniques}

\section{Patient}

A 75-year-old man was referred to our hospital because of an abnormality on a sputum examination. Chest computed tomography showed a $37 \times 20 \mathrm{~mm}^{2}$ lung tumor in the right $\mathrm{S} 6$ widely adjacent to the membranous portion of the intermediate bronchus (Figure 1A,B). Endobronchial ultrasound transbronchial needle aspiration revealed adenocarcinoma without evidence of nodal metastasis. The clinical diagnosis was a stage IB (T2aN0M0) adenocarcinoma, and surgical treatment was indicated.

\section{Surgical procedure}

A schematic illustration of the operation is shown in Figure 2. Due to the extensive tumor location along the intermediate bronchus, right lower sleeve lobectomy with double-barrel bronchoplasty of the right upper and middle lobar bronchi was preoperatively planned. During the operation, however, the tumor was noted to further extend into the right upper bronchus and S2 from the intermediate bronchus (Figure $3 A$ ), and right upper lobectomy was considered to be inevitable for complete resection of the tumor. Intending to the balance of oncological curability and a preserved pulmonary function, the initial plan was changed to sleeve resection of the right upper lobe and S6.

The right main bronchus (RMB), MLB and BB were carefully exposed and transected to obtain sufficient surgical margins (Figure 3B,C), and a new bifurcation between the MLB and BB was created. The new orifice was then anastomosed to the RMB in the double-barreled fashion. After trimming the bronchial edges, MLB and BB were combined with three interrupted sutures of 4-0 polydioxanone sutures (PDSII, Johnson \& Johnson, Tokyo, Japan) in order to make a combined orifice obtuse triangle (Figure 4A). These three sutures were ligated inside the bronchial lumen to secure a tight knot. Prior to docking the combined bronchi to the RMB, the membranous portion of the RMB stump was shorten by a stich with 4-0 PDSII to adjust to the orifice of the combined bronchi (Figure 4B). The pericardium was dissected below the inferior pulmonary vein and a folded sterilized gauze was placed in the space under the middle lobe and basilar segment to relieve tension between the anastomosis. End-to-end anastomosis in a double-barrel fashion was performed with continuous running sutures in most of the membranous portion of the RMB and nine interrupted 

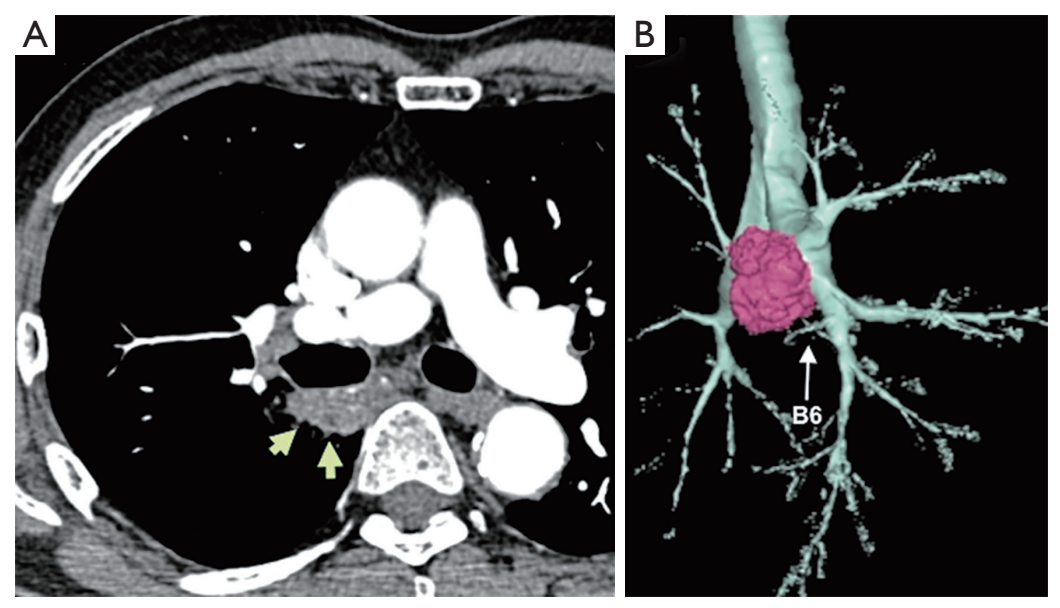

Figure 1 Preoperative chest computed tomography. (A) The axial view showed the tumor in the right S6 (yellow arrow) was adjacent to the membranous portion of the intermediate bronchus; (B) a three-dimensional view showed that the tumor extended to near the orifice of the right upper bronchus.
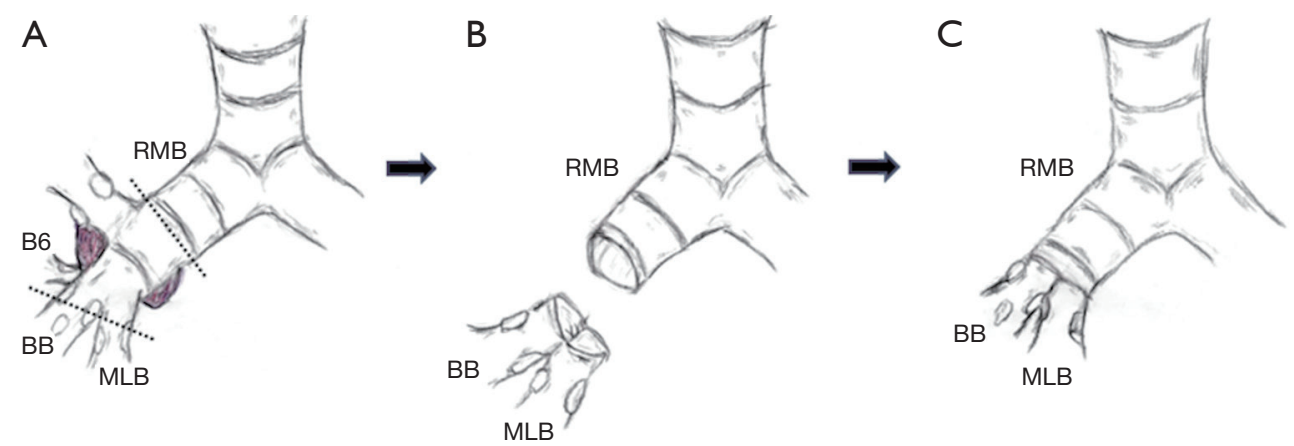

Figure 2 Schematic illustration of the operative procedure. (A) Bronchial sleeve resection of the right upper lobe and S6 was performed. The middle lobar bronchus (MLB) and basilar bronchus (BB) were transected to obtain a sufficient surgical margin; (B) a new bifurcation of the MLB and BB was created; (C) the MLB and BB were anastomosed to the right main bronchus in a double-barrel fashion.
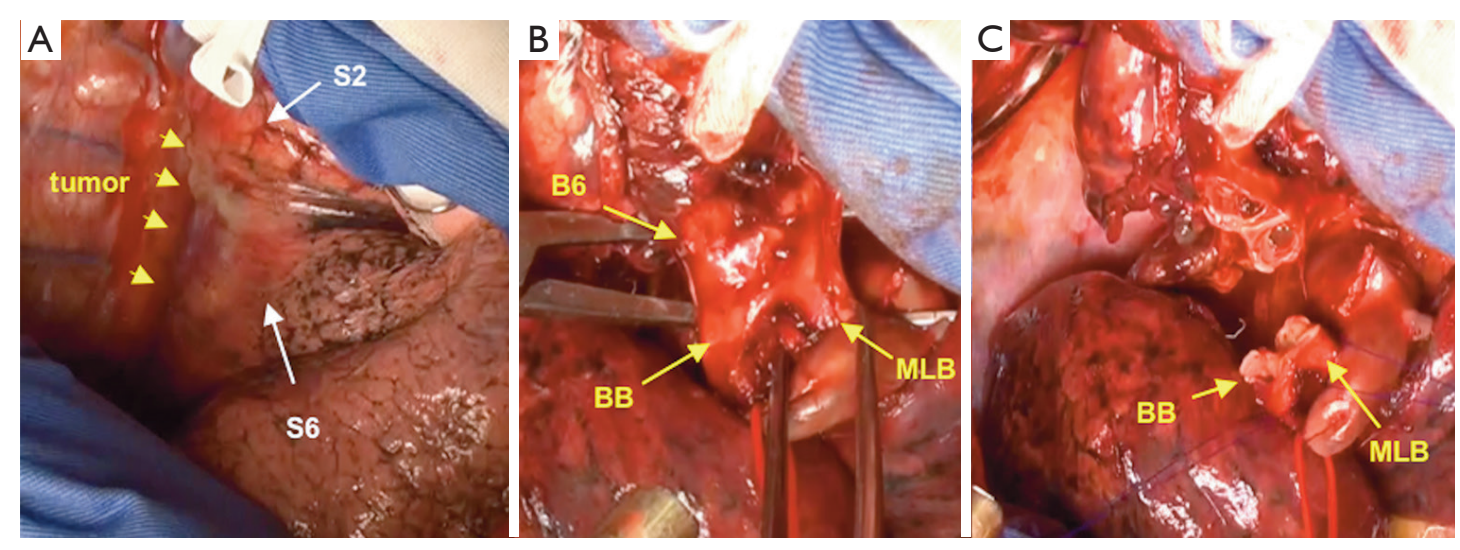

Figure 3 The intraoperative findings of a tumor that required bronchial resection.(A) The tumor extended into the right upper bronchus and S2 from the intermediate bronchus; $(\mathrm{B}, \mathrm{C})$ the right main bronchus (RMB), MLB and BB were carefully transected to obtain sufficient surgical margins. 

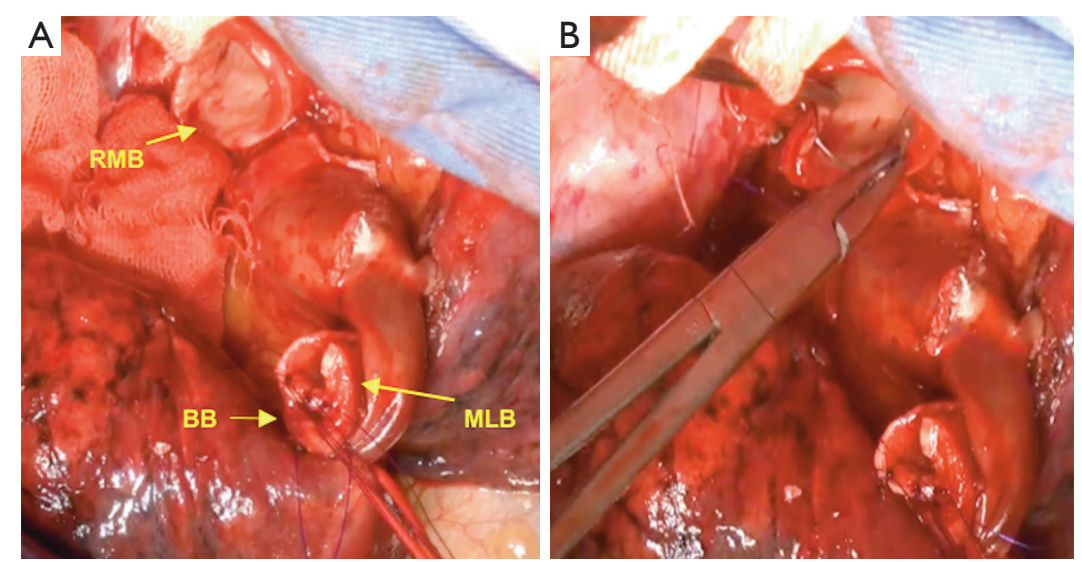

Figure 4 Adjusting each orifice of the RMB, MLB and BB. (A) The MLB and BB were combined with three interrupted sutures; (B) the membranous portion of the RMB stump was shortened by a stich to adjust to the orifice of the combined bronchi. RMB, right main bronchus; MLB, middle lobe bronchus; BB, basilar bronchus.

sutures at other sites with 4-0 PDSII (Figure 5). In the cartilage portion, some of the interrupted sutures at the deep site were ligated inside the lumen or otherwise ligated outside. The anastomosis was wrapped with the pericardial fat (Figure 5D). Pulmonary arterial reconstruction was waived because the pulmonary artery didn't kink after the bronchoplasty. The surgical procedure is described in detail in the video file (Figure 6). The final diagnosis was p-T2aN2M0, stage IIIA. A pathological examination confirmed that the cut line of the bronchi was made at a site that was sufficiently separated from the main tumor.

The postoperative course was uneventful without any complications. Postoperative bronchoscopy presented with satisfactory healing and patency (Figure 7). The postoperative forced vital capacity (FVC), forced expiratory volume in the first second (FEV1.0), and FEV1.0/FVC at 5 months after surgery were $2.01,1.41 \mathrm{~L}$, and $70.1 \%$, respectively, whereas preoperatively they had been 2.73 , $2.00 \mathrm{~L}$, and $73.3 \%$, respectively.

\section{Comments}

ESL is feasible, with perioperative complication rates similar to those of conventional sleeve lobectomies, and various types of resection have been applied according to tumor location $(1,2)$. Double-barrel reconstruction is sometimes selected in cases requiring the reconstruction of lobar bronchi and proven its feasibility (5-7); however, peripheral bronchoplasty, at segmental bronchus level, with double-barrel reconstruction is rarely reported.
Double-barrel reconstruction is classified into three types based on previous reports, which included cases of carinal resection. Type A (carinal reconstruction): the bilateral main bronchi is anastomosed to the trachea to create a new carina (8). This is the most common use of double-barrel bronchoplasty. Type B: the lobar bronchi is anastomosed to the main bronchus [e.g., the right upper lobar bronchus and the MLB or intermediate bronchus, the right lower lobar bronchus and the MLB, and the left upper and lower lobar bronchus (5-7)]. Type C: the main bronchus is anastomosed more peripherally at the segmental bronchus level [e.g., the MLB and BB, and the left B6 and BB (9)]; our case was classified as type $C$. There are some surgical tips to be considered during each type of double-barrel bronchoplasty. First, the size of the anastomosed bronchial lumen differs according to the location of anastomosis. The anastomosed lumen becomes narrower in the peripheral regions, especially in the segmental bronchi. Trimming the bronchial stumps is important for maintaining the patency of the lumen because the protrusion of the bronchial cartilage into the lumen can cause postoperative stenosis at the site of anastomosis. This point should be especially notified when bronchial reconstruction is performed at the periphery, such as in type $\mathrm{C}$ procedures. Second, anastomotic deformation due to dislocation and/or torsion of the preserved lobes/ segments can lead to critical problems and should be avoided. Attention should always be paid to ensure that the site of anastomosis is in a natural position in each lobe/segment, without considering each membranous or cartilage part of the bronchus in type $\mathrm{B}$ and $\mathrm{C}$ procedures (6). 

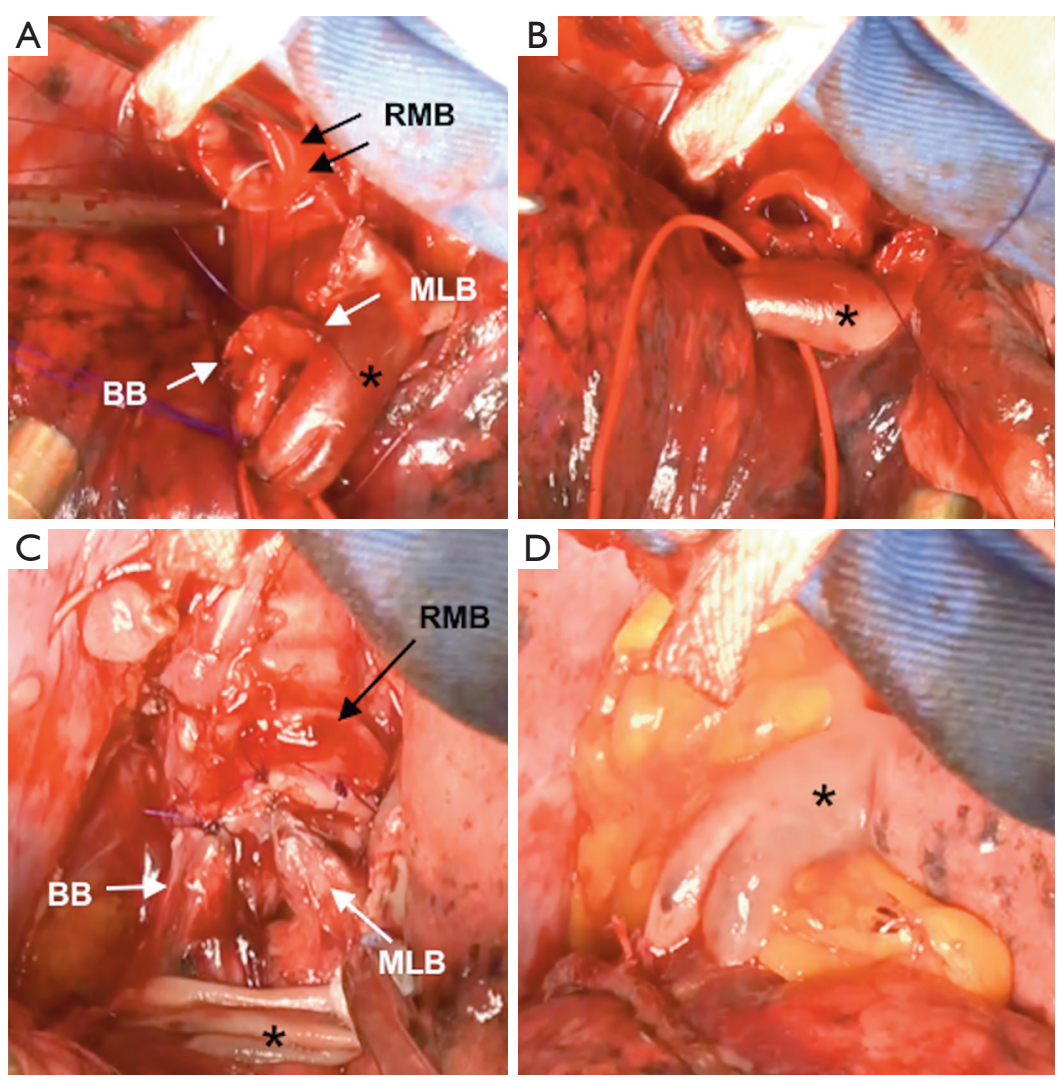

Figure 5 End-to-end anastomosis in a double-barrel fashion. (A) Interrupted sutures were performed at the deep anastomotic site; (B) interrupted sutures were placed at the shallow cartilage portion of the anastomotic site; (C) segmental bronchoplasty was successfully performed in a double-barrel fashion; (D) the anastomotic site was wrapped with pericardial fat to separate the site of anastomosis from the pulmonary artery. The asterisk in each figure indicates the basal trunk of the pulmonary artery.

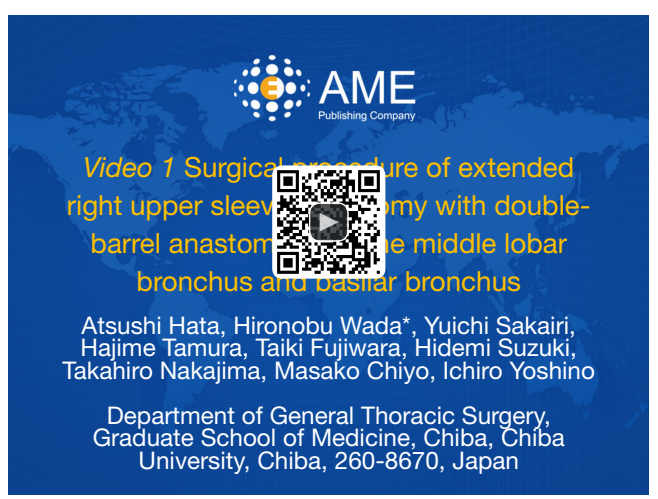

Figure 6 Surgical procedure of extended right upper sleeve lobectomy with double-barrel anastomosis of the middle lobar bronchus and basilar bronchus (4). MLB, middle lobe bronchus; $\mathrm{BB}$, basilar bronchus.

Available online: http://www.asvide.com/article/view/24996
Third, the blood supply for anastomosis differs according to the level of anastomosis. The blood supply of the lower trachea and main bronchi comes from the branch of inferior thyroid, subclavian, superior intercostal, and bronchial arteries (10); however, the blood supply of the lobar and segmental bronchi mainly comes from the pulmonary circulation (up to $75 \%$ to $90 \%$ ). Thus, for type A procedures, there is generally little dissection of the membranous portion in order to preserve the blood supply from the bronchial arteries and protect the site of anastomosis. On the other hand, for type B and C procedures, distal anastomosis near the pulmonary parenchyma has an advantage in that the blood supply comes from pulmonary circulation. Double-barrel reconstruction of the segmental bronchi receives the greatest blood supply benefit as the site of anastomosis is closest to the lung parenchyma.

There are also some common surgical tips for all types 


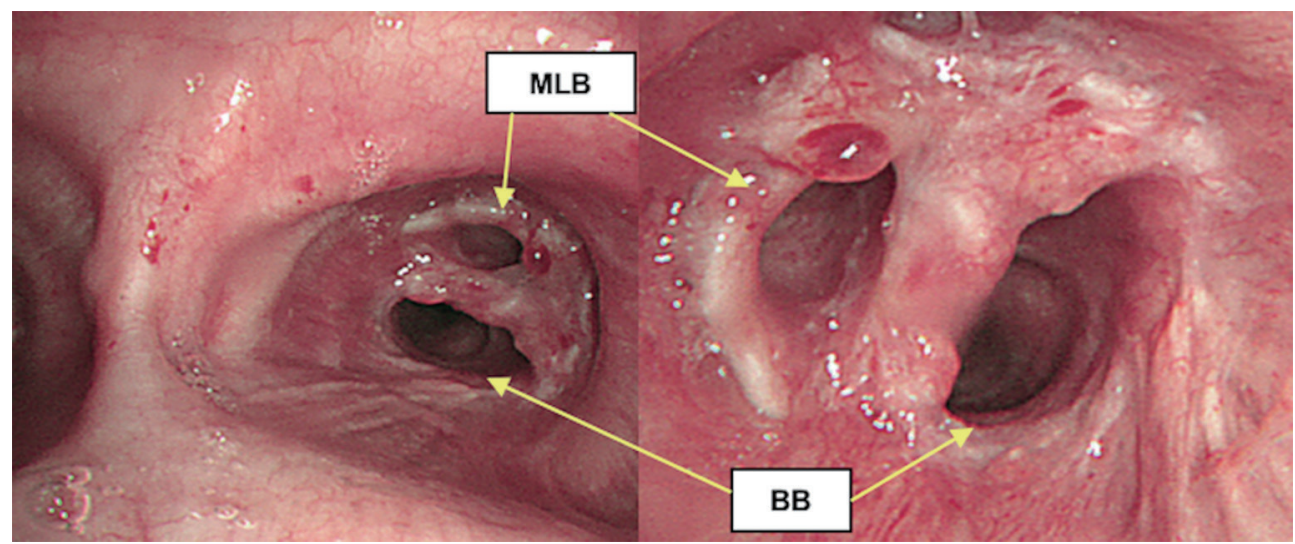

Figure 7 Postoperative bronchoscopy at seven months after surgery. RMB, right main bronchus; MLB, middle lobe bronchus; BB, basilar bronchus.

of double-barrel reconstruction. First, we attempt to make the combined orifice obtuse triangle with internal knotsutures on the distal side. This can be matched well to the horseshoe-shaped orifice on the proximal side. In carinal reconstruction, external knot-suture is used for the top of new bifurcation in order to keep the two distal bronchi in the appropriate position. Second, all types of reconstruction require tension release, this is achieved by the incision of the pericardium beneath the hilum and the placement of a folded sterilized gauze under the remaining lobes or segments. Tension at the site of anastomosis is undesirable when performing double-barrel reconstruction.

ESL with double-barrel reconstruction at the segmental bronchi has some advantages. First, this technique yields sufficient surgical margins from the tumor for complete resection. Okada et al. postulated that a margin should be at least $1 \mathrm{~cm}$ apart from the macroscopically tumorfree bronchus (1). The extended tumor location of the current case supported the application of double-barrel segmental bronchoplasty. Second advantage is that bronchial anastomosis is made at the periphery. Since distal bronchial circulation is supplied by the peripheral lung parenchyma as we described above, the anastomosis can heal better. Third, this approach is advantageous, from the viewpoint of the residual pulmonary function, compared with right pneumonectomy or bilobectomy of the upper and lower lobes. Indeed, the present patient's post-operative pulmonary function was well preserved and almost identical to the predicted value.

However, preserving a basilar segment is oncologically controversial in cases of lung carcinoma originating in the S6. In our case, the operative procedure was decided for several reasons. First, right upper and lower bilobectomy left only the middle lobe remaining, which can be a hazardous condition due to potential kinking and twisting of the hilar structures. Second, the tumor was mainly located in the superior part of the S6 with sufficient distance from the basilar segment. Finally, the loss of a large amount of lung parenchyma is a disadvantage for not only the quality of life but also additional post-operative treatments.

We herein reported a case of sleeve resection of the right upper lobe and S6 with reconstruction in a double-barrel manner. This novel technique can be applied for centrally located tumors in the superior segment in the right lower lobe in order to preserve the pulmonary function.

\section{Acknowledgements}

None.

\section{Footnote}

Conflicts of Interest: T Nakajima and I Yoshino received honoraria and/or lecture fee from Johnson \& Johnson and Olympus Medical Systems. Others authors have no conflicts of interest to declare.

Informed Consent: Written informed consent was obtained from the patient for publication of this manuscript and any accompanying images.

\section{References}

1. Okada M, Tsubota N, Yoshimura M, et al. Extended 
sleeve lobectomy for lung cancer: the avoidance of pneumonectomy. J Thorac Cardiovasc Surg 1999;118:7103; discussion 713-4.

2. Chida M, Minowa M, Miyoshi S, et al. Extended sleeve lobectomy for locally advanced lung cancer. Ann Thorac Surg 2009;87:900-5.

3. Berthet JP, Paradela M, Jimenez MJ, et al. Extended sleeve lobectomy: one more step toward avoiding pneumonectomy in centrally located lung cancer. Ann Thorac Surg 2013;96:1988-97.

4. Hata A, Wada H, Sakairi Y, et al. Surgical procedure of extended right upper sleeve lobectomy with double-barrel anastomosis of the middle lobar bronchus and basilar bronchus. Asvide 2018;5:523. Available online: http://www. asvide.com/article/view/24996

5. Jiang $\mathrm{X}$, Dong $\mathrm{X}$, Zhao $\mathrm{X}$, et al. Bronchial sleeve resection distal to the main bronchus with complete pulmonary preservation for benign or low-grade malignant tumors.

Cite this article as: Hata $\mathrm{A}$, Wada $\mathrm{H}$, Sakairi $\mathrm{Y}$, Tamura H, Fujiwara T, Suzuki H, Nakajima T, Chiyo M, Yoshino I. Extended right upper sleeve lobectomy with double-barrel segmental bronchoplasty. J Thorac Dis 2018;10(5):3059-3064. doi: $10.21037 /$ jtd.2018.04.135
Ann Thorac Surg 2007;84:e19-21.

6. Tanaka K, Nakajima T, Morimoto J, et al. Right lower sleeve lobectomy with double-barreled bronchoplasty for a centrally located lung cancer. Ann Thorac Surg 2015;99:1829.

7. Tamura M, Oda M, Matsumoto I, et al. Double-barrel reconstruction for complex bronchial disruption due to blunt thoracic trauma. Ann Thorac Surg 2009;88:2008-10.

8. Macchiarini P, Altmayer M, Go T, et al. Technical innovations of carinal resection for nonsmall-cell lung cancer. Ann Thorac Surg 2006;82:1989-97; discussion 1997.

9. Uramoto H, Nakajima Y, Kinoshita H. Left upper lobe double sleeve lobectomy with double barrel anastomosis for lung adenocarcinoma. Ann Med Surg (Lond) 2016;9:12-4.

10. Fréchette E, Deslauriers J. Surgical anatomy of the bronchial tree and pulmonary artery. Semin Thorac Cardiovasc Surg 2006;18:77-84. 